\title{
ESTUDIOS MORFOMÉTRICOS APLICADOS A PUNTAS DE PROYECTIL LANCEOLADAS DEL HOLOCENO TEMPRANO-MEDIO EN SIERRAS Y LLANURAS PAMPEANAS DE ARGENTINA
}

\author{
Guillermo Heider y Diego Rivero
}

\begin{abstract}
En este trabajo exponemos y discutimos los resultados obtenidos a partir de estudios realizados a puntas de proyectil lanceoladas recuperadas en sectores acotados de las sierras y llanuras pampeanas de Argentina. El análisis se basó en puntos morfométricos adicionando, además, variables como largo, ancho, espesor y materia prima de los especímenes. La morfometría geométrica fue la principal herramienta utilizada, complementada por estudios tipológicos clásicos y estadística multivariada. Los instrumentos líticos examinados, pertenecientes a sistemas de armas utilizados por cazadores-recolectores, son comúnmente identificados como puntas Ayampitín. Estos constituyen un elemento diagnóstico sobre la tecnología existente en el centro de Argentina durante el Holoceno temprano-medio. Los resultados permitieron discutir que a la variabilidad propia del conjunto se adiciona una variabilidad interna en los tipos morfológicos básicos (pedunculadas y apedunculadas). Además, la selección de rocas para la talla estuvo fuertemente ligada a las aptitudes físicas de aquellas, existiendo, asimismo, una correlación entre algunas materias primas y las formas de los ejemplares. Finalmente, los diseños básicos fueron utilizados en un sistema de propulsor. La discusión permitió plantear nuevas líneas de evidencia a desarrollar para aportar al conocimiento de las etapas iniciales del poblamiento sudamericano.
\end{abstract}

In this article we present and discuss the results of a study of lanceolate projectile points recovered from sectors of the Pampean Mountains and Plains of Argentina. This morphometric analysis was based on the length, width, thickness, and raw materials of the specimen. Geometric morphometry was complemented with classical typological studies and multivariate statistics. The lithic tools examined, known as ayampitín points, correspond to a weapon system used by Early-Mid Holocene hunter-gatherers in Central Argentina. The results indicate that there is an internal variability in the basic morphological types (stemmed vs. unstemmed), in addition to the ensemble's own variability. Furthermore, the rocks selected for knapping were based on their aptitude, demonstrating a correlation between raw materials and the forms of the specimens. Finally, these projectile points were used as darts propelled by atlatls. The discussion raises new lines of evidence that may be pursued to develop more knowledge on the initial stages of the peopling of South America.

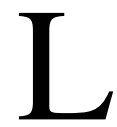

as puntas de proyectil lanceoladas formaron parte de sistemas de armas presentes en una significativa macrorregión de Sudamérica durante diferentes momentos del Holoceno temprano y medio. La importancia de este tipo de diseños ha sido planteada por investigadores que trabajaron con distintos enfoques teóricos, escalas espaciales y metodologías de estudio (Aschero et al. 2011; De Souza 2006;
Hocsman 2010; Martínez 2007; Ratto 2006). Si bien no se ha puesto el acento de manera específica sobre la distribución espacial de esta tipología de puntas, las mismas son comunes en diferentes porciones andinas del Noroeste y Cuyo en Argentina, en el norte y centro de Chile además del sur de Bolivia (Capriles y Albarracín-Jordán 2013; Cardillo 2006; Hoguin y Yacobaccio 2012; Jackson et al. 2011; Martínez

\footnotetext{
Guillermo Heider a CONICET-CCT San Luis; IFDC-San Luis; Departamento de Geología, Facultad de Ciencias Físico Matemáticas y Naturales, Universidad Nacional de San Luis, Avenida Ejército de Los Andes 950, San Luis, Argentina (guillermoheider@hotmail.com).

Diego Rivero " IEH-CONICET "Prof. Carlos S. A. Segreti”; Cátedra de Prehistoria y Arqueología, Facultad de Filosofía y Humanidades, Universidad Nacional de Córdoba, Vía de las Humanidades s/n, Córdoba, Argentina (ayampitin1@yahoo.com.ar).
}

Latin American Antiquity 29(3), 2018, pp. 572-590

Copyright ( 2018 by the Society for American Archaeology doi:10.1017/laq.2018.20 


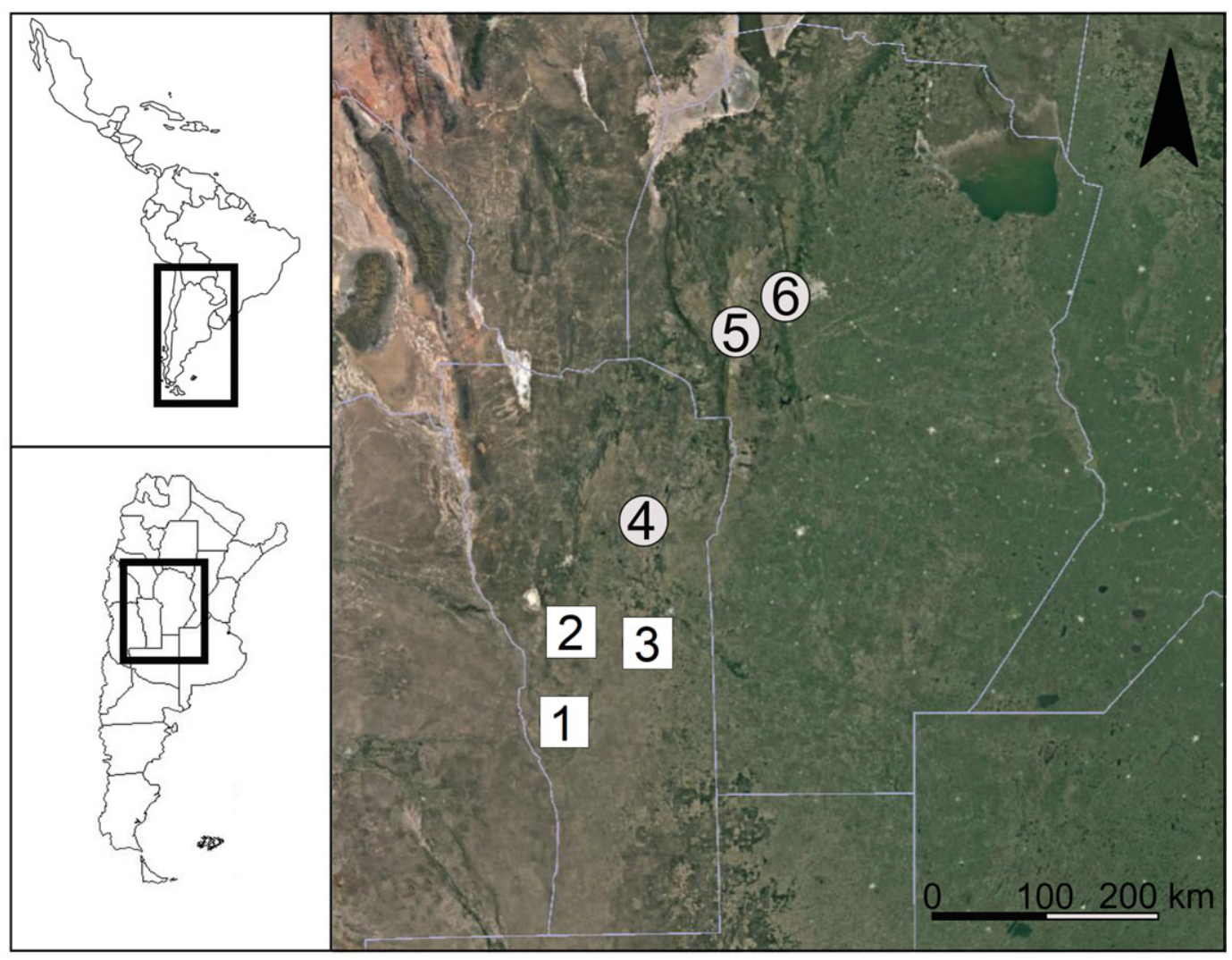

Figura 1. Ubicación de los sectores en donde se recuperaron las puntas de proyectil analizadas: 1) Cruz del Sur y La Iseca; 2) Alto Pelado; 3) Lavaisse y Sayape; 4) Planta de Sandía; 5) Pampa de Achala; 6) San Roque. Los números enmarcados en cuadrado corresponden al norte de Pampa Seca, en círculo corresponden a las Sierras Centrales. (Color online)

2003; Núñez et al. 2005; Ratto 2003; Restifo 2013). Las sierras pampeanas constituyeron, hasta no hace mucho tiempo, un límite tanto meridional como oriental para la distribución de puntas de proyectil lanceoladas. Recientes investigaciones mostraron la presencia de un número significativo de cabezales líticos con este diseño en un sector ubicado en la planicie sur contigua, en el noroeste de la región pampeana argentina (Heider 2015).

En este trabajo se estudia un conjunto de puntas de proyectil lanceoladas procedentes del sur de las sierras pampeanas (comúnmente denominadas Sierras Centrales) y del norte de Pampa Seca (en el noroeste de la región pampeana argentina; Figura 1). La particularidad del abordaje aplicado corresponde a las metodologías utilizadas. Además de la caracterización tecno-morfológica tradicional de los artefac- tos, estas incluyen la morfometría geométrica y la estadística multivariada (Bookstein 1982; Mitteroecker y Gunz 2009; Torcida y Perez 2012). En este sentido, si bien estudios similares al que presentamos aquí fueron aplicados de manera profusa en algunas regiones de Sudamérica como el Noroeste y Patagonia en Argentina, el sur de Brasil o las llanuras de Uruguay (Cardillo 2006, 2010; Cardillo y Charlín 2010; Castiñeira et al. 2011; Charlin y Gónzalez-José 2012; Franco et al. 2009; Okumura y Araujo 2014), en el sector aquí considerado esta metodología ha sido escasamente aplicada (Rivero y Heider 2017). De esta forma, se pretende contribuir de manera general a las discusiones sobre la tecnología de armas arrojadizas y el poblamiento en el centro y el oeste de Argentina, mediante metodologías de análisis novedosas para la región, utilizando una escala temporal 
amplia que abarca la parte final del Holoceno inicial y todo el Holoceno medio.

Es notorio el contraste, en cuanto a cantidad de puntas recuperadas, entre las Sierras Centrales y la región pampeana. Los diseños lanceolados tienen abundante presencia en sitios superficiales, de estratigrafía, colecciones y bibliografía arqueológica del área serrana (e.g., González 1960; Pautassi 2011; Rivero 2009). En contraposición, en la región pampeana solo fueron registradas en los niveles intermedios de Casa de Piedra (provincia de La Pampa) y en Campo Laborde (provincia de Buenos Aires) donde se recuperó una pieza fracturada (Gradín 1984; Politis et al. 2004). Los estudios realizados recientemente en el noroeste de la región pampeana permitieron revertir parcialmente este panorama al registrar 77 puntas lanceoladas, incluyendo los especímenes fracturados (Heider 2015).

En la región del centro de Argentina, los cabezales líticos lanceolados son denominados de manera genérica como puntas Ayampitín. Estos se encuentran relacionados, desde la creación de la secuencia maestra de Intihuasi (González 1960), a momentos tempranos de la presencia humana en las provincias de San Luis y Córdoba, Argentina (Pautassi 2011; Rivero 2009; Sario 2008). Sin embargo, el término se encuentra aún hoy fuertemente ligado (en ámbitos académicos y no académicos) a la "cultura Ayampitín" (González 1960). En este sentido, actualmente el concepto responde tanto a una tradición tecnológica general, como a los cabezales líticos de forma lanceolada distribuidos en un territorio mayor a los $100.000 \mathrm{~km}^{2}$ del centro argentino, enmarcados, además, en una temporalidad mayor a los 2.000 años a partir de finales del Holoceno temprano (Rivero 2009). Particularmente las puntas Ayampitín son, aún hoy, utilizadas como elementos temporalmente diagnósticos para caracterizar diferentes momentos del poblamiento regional (Laguens et al. 2007; Rivero 2012). Como grupo tipológico han sido objeto de estudios tecnológicos referidos a procesos de manufactura como así también en relación a su empleo en estrategias de caza (Pautassi 2011; Rivero y Heider 2016; Sario 2008).

En definitiva, este trabajo se plantea como un aporte para la discusión macrorregional de dos líneas de notoria actualidad: la tecnología de cabezales líticos como parte de sistemas de armas y las etapas de poblamiento del centro de Argentina. Un punto de partida es la revisión de las descripciones existentes para los cabezales líticos Ayampitín mencionadas para el centro de Argentina, las cuales son exiguas en comparación con otras regiones del país y Chile. Son objetivos centrales, por una parte, abordar la variabilidad existente dentro del universo de puntas lanceoladas del sector central de Argentina, desde la morfometría geométrica y la estadística multivariada, definiendo paralelamente los posibles sistemas de armas empleados. En este sentido, se evalúa cómo influyen en la variabilidad observada los sistemas de armas, la materia prima y las actividades de mantenimiento o reactivación de los cabezales líticos. Por otra parte, se busca aportar a la discusión macrorregional sobre el proceso de poblamiento, insertando la discusión regional y sus particularidades en un contexto más amplio. Para alcanzar los objetivos planteados se utilizaron como universo muestral todas las puntas de proyectil que estuvieran enteras y presentaran tipología lanceolada (sensu Aschero 1975) recuperadas en sitios arqueológicos o presentes en colecciones que procedan de las sierras y llanuras pampeanas. En relación con esto, y debido a las necesidades de los métodos utilizados, en esta instancia de investigación fueron descartadas más de 500 puntas de proyectil fracturadas con idénticas procedencias.

\section{Las Puntas de Proyectil Ayampitín como Caso de Estudio}

La representación de secuencias maestras, complejos culturales o grupos sociales ha estado permanentemente ligada al uso de puntas de proyectil como artefactos temporalmente sensitivos y con significación histórica en áreas geográficas particulares (Bettinger et al. 1991; Knecht 1997; Martínez 2003; Ratto 2003; Rondeau 1996). Sin embargo, diversos autores explicitan dudas sobre la construcción de tipologías para su uso como "fósiles guía" o aportes a secuencias maestras (Martínez 2003). En el caso del estudio que se presenta, se considera válida la utilización de la cronología relativa a partir de puntas de proyectil para abordar las problemáticas propuestas y su 
posible relación con contextos espaciales y temporales a nivel regional y macrorregional (Rivero 2012).

Las ocupaciones iniciales para el centro de Argentina fueron propuestas, por diversos investigadores, para momentos finales del tardiglaciar entre aproximadamente 11.000 y 9300 años aP (Laguens et al. 2007; Rivero 2012; Sario 2011). Las evidencias sobre este período son aún escasas, tanto en superficie como en estratigrafía. En este sentido, solo se han registrado puntas de proyectil Cola de Pescado o Fell 1 fracturadas, las cuales constituyen un elemento diagnóstico del tardiglaciar en Sudamérica (Cattaneo et al. 2016; Rivero et al. 2015). Además, los contextos con dataciones absolutas se restringen a los componentes inferiores de los sitios de El Alto 3 (11.010 \pm 80 aP [LP-1506; madera carbonizada] y $9790 \pm 60$ aP [LP-1420; madera carbonizada]) y la Gruta de Candonga ( $10.450 \pm 50 \mathrm{aP}$ [SRLA1062; hueso humano]), ambos localizados en las sierras de Córdoba (Cornero et al. 2014:290; Rivero 2009:54). En el noroeste de la región pampeana, en contraposición a lo que ocurre para las Sierras Centrales, sólo se cuenta con un fechado radiocarbónico de finales del Holoceno temprano sin asociación contextual con puntas de proyectil (Balbuena et al. 1982).

Con posterioridad al periodo inicial de poblamiento se observa un aumento de la señal arqueológica regional, aproximadamente a partir de 8000 aP (Rivero 2009, 2012). Recientemente se propuso que, a partir de fines del Holoceno temprano, ocurrió una re-colonización y ocupación efectiva de los diferentes sectores del centro de Argentina (Rivero 2012). En contraposición a esto, Laguens y colaboradores (2007) proponen una posible continuidad ocupacional desde el momento inicial de exploración. Aún ante esta discusión, las puntas de proyectil lanceoladas o Ayampitín tienen consenso como elemento tecnológico temporalmente diagnóstico para finales del Holoceno inicial y parte del Holoceno medio. Inicialmente su presencia fue temporalmente acotada por González (1960) al crear la primera secuencia cronológico-cultural macrorregional. A partir de esa publicación las puntas Ayampitín han sido utilizadas para contextualizar cronológicamente conjuntos arqueológicos no datados y para la discusión sobre la temporalidad de la presencia humana en las Sierras Centrales (Pautassi 2011; Rivero 2009; Sario 2008). Más aún, la mayor parte de los contextos datados en esta región (i.e., componentes tempranos de los sitios de Intihuasi, El Alto 3, Arroyo El Gaucho 1 y Quebrada del Real 1) poseen como artefacto característico las puntas de proyectil lanceoladas, que se han datado en un rango que va entre aproximadamente 8500 y 6000 años aP (González 1960; Rivero 2009, 2012).

A nivel macrorregional, se han registrado cabezales líticos con tipologías lanceoladas en el norte y centro de Chile, la Puna y otros sectores andinos de Argentina. Es notorio que la observación inicial sobre la presencia de puntas lanceoladas en una escala macrorregional fuera tempranamente hecha por González (1952, 1960), quien sostiene una relación directa del poblamiento con procesos de migración de origen andino, vinculado con una tradición panandina de puntas de proyectil lanceoladas de dispersión continental desde Colombia al centrooeste Argentino. Más recientemente la importancia de las mismas fue planteada desde diferentes miradas teórico-metodológicas y con diversas escalas temporales y espaciales (Cardillo 2002; Hoguin y Yacobaccio 2012; Martínez 2003; Pintar 2004; Ratto 2006; Restifo 2013). Un análisis más detallado, orientado a los sectores cercanos de Cuyo, permite realizar una calibración temporal relativa para este tipo de diseños. La cueva de El Peñoncito, ubicada en el norte de la provincia de San Juan, presenta en su Nivel I puntas de proyectil lanceoladas con dataciones en torno a los 7500 y 7000 aP (Berberián y Calandra 1984). Para el suroeste de esta provincia se realizó una clasificación tipológica de este tipo de puntas, a la que se asignaron temporalidades similares, en Los Morrillos y La Colorada de La Fortuna (Gambier 1974). García (2003, 2010) menciona contextos similares en Gruta del Manzano y en registros superficiales de los valles de Uspallata y Uco (provincia de Mendoza), además de la precordillera. Menos clara es la situación en el centro y sur de Mendoza; allí las discusiones giran actualmente en torno a los factores causales de la disminución del registro arqueológico para el periodo. Finalmente, es pertinente incluir en esta mención de tecnologías y contextos similares a Ayampitín en Cuyo el complejo Huentelauquén 
del centro de Chile. En este sentido, es de notoria actualidad la propuesta de una interacción y movimientos de bandas a escala macrorregional con vinculación a ambas vertientes cordilleranas (Bárcena 2001; Cortegoso 2005, 2014; Durán 1997; García 2005; Jackson et al. 2011; Neme y Gil 2009).

En definitiva, como puede observarse, un importante número de cabezales líticos con morfologías lanceoladas presentan cronologías y localización geográfica acotadas, como así también un nombre característico (e.g., puntas San Martín, La Fortuna, Quebrada Seca, El Perchel y Peña de la Cruz). La descripción inicial de las puntas Ayampitín señala que su limbo es “... de forma lanceolada o de hojas de laurel o almendra, con un largo que varía entre $45 \mathrm{~mm}$ y $100 \mathrm{~mm}$. $\mathrm{El}$ ancho oscila entre 16 y $24 \mathrm{~mm}$ y la base es siempre semicircular. Por lo general son bastante espesas o de sección oval y más frecuentemente en diamante. Están trabajadas en ambas caras por retoque y presión" (González 1960:108). El autor distingue, además, un conjunto de variaciones o asimetrías siendo la más importante la presencia de pedúnculo: “... son idénticas a las típicas, pero en vez de tener la base perfectamente semicircular presentan una asimetría apenas marcada que interrumpe la línea regular del borde para adentrarse hacia el centro de la pieza por medio de una serie de pequeños retoques" (González 1960:108). Recientemente fueron adicionados a esta definición nueva terminología y características de manufactura surgidas de la talla experimental (ver Pautassi 2011). A excepción de los trabajos aquí mencionados, no se cuenta con estudios de detalle sobre las características centrales de la tipología Ayampitín. Por esto, aquí utilizamos una definición tipológica clásica como punto de partida, entendiendo entonces los cabezales líticos como artefactos de forma geométrica de contorno lanceolado, limbo subparalelo, sección transversal biconvexa simétrica, bordes de limbo normal y posible presencia de pedúnculo (esbozado o destacado). Tecnológicamente son piezas confeccionadas mediante adelgazamiento bifacial con lascados escamosos extendido irregular o paralelo transversal con forma base en piezas bifaciales o lascas de arista grandes o muy grandes. Aún ante esta descripción, nos parece pertinente mencionar que, si bien todas las puntas analizadas se inscriben dentro de una forma lanceolada (sensu Aschero 1975) esta denominación no alcanza a captar las diferencias observadas. Por ello, la distinción de posibles patrones técnico-estilísticos es uno de los objetivos del trabajo, utilizando para ello la morfometría geométrica y la estadística multivariada como herramienta.

\section{Los Ámbitos Geográficos de Recuperación de la Muestra}

Las puntas de proyectil analizadas (Figura 2) fueron recuperadas en el contexto de dos áreas geográficas de la región central de Argentina, la primera centrada inicialmente en las pampas de altura de las Sierras Centrales (específicamente Pampa de Achala, Córdoba) y la segunda en el norte de Pampa Seca (sur de San Luis y Córdoba; Heider 2015; Rivero 2009). Durante el trabajo de campo se utilizaron metodologías propias de la arqueología distribucional (sensu Ebert 1992). El material recolectado fue analizado siguiendo las propuestas de Aschero (1975) y Aschero y Hocsman (2004). Sin embargo, las diferencias entre ambos sectores son notorias a dos niveles: las características ambientales (climáticas, ecológicas, geomorfológicas y geológicas) y la cantidad de investigaciones realizadas a lo largo del tiempo (con un desarrollo más extenso a favor del sector serrano). Esto último determina diferencias importantes en cuanto al número de sitios arqueológicos con estratigrafía analizados y datados radiocarbónicamente (Cattáneo et al. 2013; Heider y Curtoni 2016).

En el panorama expuesto, las puntas de proyectil pueden ser subdivididas de acuerdo al ámbito geográfico de recuperación (Figura 1). El primer subconjunto incluye las puntas provenientes del norte de Pampa Seca, un paisaje llano y con alturas menores a $300 \mathrm{~m}$ snm en el sur de la provincia de San Luis (Demaría 2008; Tripaldi y Forman 2007). El segundo subconjunto fue obtenido en paisajes serranos, con pampas de altura, valles y quebradas emplazados a más de $700 \mathrm{~m}$ snm en las Sierras Centrales de San Luis y Córdoba, perteneciente al sistema de las Sierras Pampeanas Occidentales.

Adicionalmente a lo ya mencionado, es notoria la variación en la oferta ambiental de rocas 

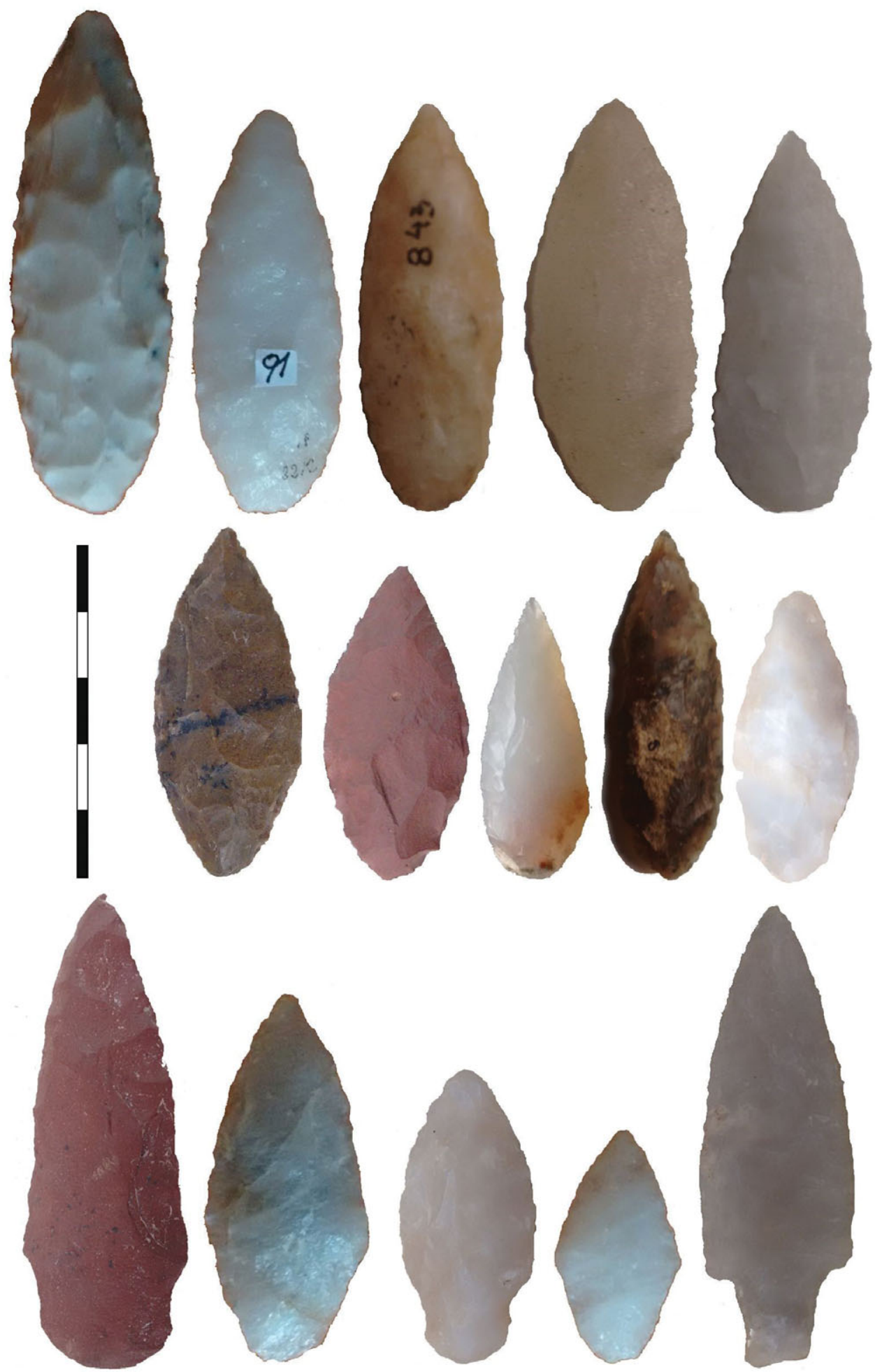

Figura 2. Ejemplos de puntas de proyectil pedunculadas y apedunculadas pertenecientes al conjunto analizado. (Color online) 
Tabla 1. Estadística Descriptiva de la Muestra de Puntas de Proyectil Analizadas.

\begin{tabular}{lcccc}
\hline & Longitud máxima $(\mathrm{mm})$ & Ancho máximo $(\mathrm{mm})$ & Espesor máximo $(\mathrm{mm})$ & Peso $(\mathrm{g})$ \\
\hline Puntas no pedunculadas & & & & \\
Media & 54.18 & 23.76 & 9.19 & 10.56 \\
Desviación estándar & 1.65 & 0.58 & 0.29 & 0.76 \\
Mediana & 50 & 23 & 8.7 & 9.3 \\
Coeficiente de variación & 28.34 & 22.77 & 29.83 & 67.07 \\
$N$ total & 87 & 87 & 87 & 87 \\
Puntas pedunculadas & 52.37 & & 8.5 & 8.77 \\
Media & 2.19 & 23.7 & 0.27 & 0.6 \\
Desviación estándar & 49.5 & 0.87 & 9 & 7.9 \\
Mediana & 27.73 & 22 & 20.74 & 45.5 \\
Coeficiente de variación & 44 & 24.40 & 44 & 44 \\
$N$ total & & 44 & & \\
\hline
\end{tabular}

aptas para la talla entre los dos sectores diferenciados dentro de las sierras y llanuras pampeanas de Argentina (Heider et al. 2015; Tabla Suplementaria 1) La Base Regional de Recursos Líticos (sensu Ericson 1984) solo fue confeccionada con detalle en todo el el norte de Pampa Seca donde se identificó una única roca de buena calidad para la talla (calcedonia) con amplia dispersión en el registro arqueológico en el centro y este de esta área (Heider y Demichelis 2015). De forma opuesta, en las Sierras Centrales la oferta de recursos puede ser considerada alta, aunque solo se realizó un estudio similar al del norte de Pampa Seca en el Valle de Conlara (provincia de San Luis). Allí, Sario (2011) localizó y caracterizó fuentes de sílice de buena calidad utilizados al menos durante el Holoceno temprano. En ambos casos, la metodología seleccionada fue el análisis petrográfico a partir de cortes de lámina delgada. Actualmente se desarrolla un estudio multi-proxy con escala macro-regional (incluyendo el norte de Pampa Seca y las Sierras Centrales) para caracterizar fuentes desde el análisis petrográfico y métodos geoquímicos, el cual cuenta ya con resultados iniciales (Heider et al. 2017).

\section{Materiales y Métodos}

El conjunto estudiado $(N=131$; Tabla 1$)$ proviene de dos ámbitos geográficos disímiles dentro de los cuales se verificaron contextos de recuperación diversos. Las investigaciones sistemáticas en ámbitos serranos permitieron recuperar ejemplares en estratigrafía correspondientes a El Alto $3(n=9)$, asociados a una datación de $7108 \pm 74$ aP (AA68145; madera carbonizada; Rivero 2009:54). El resto del subconjunto de las Sierras Centrales proviene de tres colecciones arqueológicas, dos de la provincia de Córdoba, la primera recuperada por un aficionado y cedida a los investigadores para su estudio $(n=22)$ y la otra depositada en el museo Numba-Charaba del municipio de Villa Carlos Paz $(n=37)$. Ambas fueron recolectadas en las márgenes del Lago San Roque (Valle de Punilla, provincia de Córdoba). La tercera fracción $(n=4)$, recobrada por un aficionado de Villa Mercedes (provincia de San Luis), fue obtenida en las cercanías del paraje Planta de Sandía, en el piedemonte oriental de las sierras de San Luis (Figura 1). El subconjunto del norte de Pampa Seca incluye solo tres puntas de proyectil recuperadas mediante recolección superficial sistemática. Las piezas restantes fueron analizadas a partir de dos colecciones. La primera incluye solo piezas recolectadas dentro de un médano de grandes dimensiones (Alto Pelado) en una propiedad rural de la zona central de la Travesía Puntana $(n=21)$. La segunda $(n=27)$ contiene piezas de diferentes médanos aislados y sistemas medanosos del este de la Travesía Puntana (Figura 1).

El primer paso metodológico fue abordar los cabezales líticos desde postulados tecno-tipológicos basados en los criterios propuestos por Aschero (1975) y Aschero y Hocsman (2004). Para la obtención de medidas formales (ancho, largo y espesor) se utilizó un calibre digital. Las 
puntas de proyectil, al ser sometidas a instancias de mantenimiento para extender su vida útil, pueden ver modificado su diseño básico incluso hasta el punto de transformar completamente el mismo (Aschero 1988; Knecht 1997; Martínez 2003). Se observaron las diferentes evidencias de talla (talla, retalla y retoque) presentes a fin de identificar las posibles etapas de la historia de vida de cada instrumento. La identificación de la materia prima y sus posibles fuentes de procedencia fue realizada de manera interdisciplinaria con los Drs. E. Baldo (CICTERRA-CONICET) y A. Demichelis (Departamento de Geología, Universidad Nacional de Río Cuarto). Con el objetivo de no intervenir los especímenes, se realizó una caracterización macroscópica de cada uno. Para ello, cada una de las instituciones mencionadas cuenta con los elementos técnicos necesarios y con una litoteca que abarca prácticamente la totalidad de las sierras pampeanas permitiendo, de este modo, la comparación de las puntas con muestras de mano previamente caracterizadas desde el punto de vista petrográfico.

Para la adscripción funcional de las puntas de proyectil se consideraron dos metodologías: la clasificación por peso y el análisis de función discriminante. Diversos estudios, uno incluso realizado en el área de interés, han propuesto al peso bruto como un indicador funcional adecuado (Fenenga 1953; Martínez 2003; Pastor et al. 2005). Entre estos, Fenenga (1953), sobre la base de una muestra cercana a los 800 casos, señala que las puntas de flecha poseen un peso máximo de 3,49 g. Por su parte, Hughes (1998) señala además que las puntas que no superan los $70 \mathrm{~g}$ serían puntas de dardo. A partir de un estudio experimental e investigaciones arqueológicas en las Sierras Centrales y Noroeste argentino se estimaron rangos de hasta $4 \mathrm{~g}$ para puntas de flecha, de 4,5 a $20 \mathrm{~g}$ para dardos y desde 20 a 70 g para lanza arrojadiza (Martínez 2003; Pastor et al. 2005). Sin embargo, el peso como factor discriminante para diferenciar sistema de armas se ha prestado a controversias (ver Ratto 2003). Por esta razón, se utilizó como metodología paralela el análisis de función discriminante mediante ecuaciones que utilizan dimensiones lineales de las puntas de proyectil (Shott 1997; Thomas 1978). En este trabajo se empleó la propuesta mejorada de Shott (1997:94) centrada en el ancho máximo de las puntas de proyectil (i.e., solución de una variable) ${ }^{1}$.

Para detectar la variación en la forma de las puntas se utilizó, en la segunda instancia de análisis, la morfometría geométrica. Esta técnica es de uso común en estudios biológicos, con una fuerte expansión en el campo de la arqueología (e.g., Cardillo y Charlin 2010; Castiñeira et al. 2011; Charlin y Gónzalez-José 2012). Su aplicación permite obtener una descripción cuantitativa de la forma (tanto en espacios bidimensionales como tridimensionales) a partir del análisis de las relaciones espaciales entre puntos morfométricos o landmarks, los cuales son geométricamente homólogos entre estructuras (Bookstein 1982; Gunz y Mitteroecker 2013; Mitteroecker y Gunz 2009; Rohlf y Slice 1990; Torcida y Perez 2012; Zelditch et al. 2004).

Para obtener los datos morfométricos se tomaron fotos digitales de todos los ejemplares a una distancia de $30 \mathrm{~cm}$, con una escala localizada en el costado izquierdo de los mismos. Las fotografías se compilaron en el programa TpsUtil (ver. 1.26; Rohlf 2004a), luego se digitalizaron los puntos morfométricos en TpsDig (ver. 1.4; Rohlf 2004b) ubicando dos landmarks en los puntos de máxima curvatura (en el ápice y el punto más alejado de éste en la base) y 10 semilandmarks por cada borde (20 en total). Bookstein (1991) diferencia tres tipos de landmarks según la evidencia de homología: los tipos I, II y III. Debido a la naturaleza ambigua del tipo III, el mismo fue reclasificado por Bookstein (1997), denominándolo como semilandmark. El empleo de los semilandmarks es adecuado cuando las estructuras en estudio poseen pocos puntos homólogos o cuando los mismos están concentrados en una región de la estructura. Esta situación puede presentarse cuando se analizan puntas de proyectil, las cuales (como es el caso de estudio que aquí se expone) pueden tener solo dos puntos homólogos o landmarks (el ápice y el punto más alejado de la base, orientados según el eje morfológico de la pieza). Por esta razón es necesario el uso de semilandmarks con el fin de describir adecuadamente la forma de estos artefactos. Debido a que los semilandmarks no son puntos homólogos, es necesario deslizarlos (sliding) para poder alinearlos y efectuar la superposición Procrustes. Para ello se siguió 
el criterio propuesto por Perez y colaboradores (2006), mediante la distancia Procrustes con respecto a la forma consenso. Para realizar el análisis Procrustes generalizado y el deslizamiento de los semilandmarks se utilizó el programa TpsRelw (ver. 1.54; Rohlf 2014c).

Para reducir la dimensionalidad de los datos (en este caso coordenadas Procrustes de las puntas de proyectil) se realizó un análisis de componentes principales. Esto permite visualizar diferencias, tendencias y agrupamientos de los especímenes analizados (Mitteroecker y Gunz 2009). Finalmente, se exploró la covariación entre la forma y otras variables de interés como el largo, espesor y peso. Para esto se emplearon las técnicas de regresión de mínimos cuadrados parciales (PLS, por sus siglas en inglés) y el análisis discriminante o de variable canónica (CVA, por sus siglas en inglés; Klingenberg y Monteiro 2005; Mitteroecker y Bookstein 2011; Rohlf y Corti 2000) las cuales fueron también aplicadas (y explicitada su metodología) en estudios similares en Argentina (Cardillo y Charlin 2010; Castiñeira et al. 2011 y Charlin y González-José 2012).

En nuestro caso de estudio se utilizaron las variables canónicas para explorar la asociación entre la forma de las puntas de proyectil y la materia prima utilizada. Para los análisis de estadística multivariada de componentes principales PLS y de variables canónicas, se utilizó el programa MorphoJ (Klingenberg 2009). Se importaron las coordenadas Procrustes y el tamaño centroide, definido como la raíz cuadrada de la suma de las distancias cuadradas de todos los puntos desde su centro de gravedad (Mitteroecker y Gunz 2009), obtenidos en el programa TpsRelw, agregándose variables no geométricas (e.g., espesor, presencia o ausencia de pedúnculo y tipo de materia prima) para los análisis multivariados mencionados.

Con el fin de evaluar la influencia de los procesos de reactivación sobre la variación morfológica observada, se realizó un análisis PLS entre las coordenadas Procrustes y el índice de reducción propuesto por Iriarte (1995). Este último está basado en la relación entre largo y espesor de las puntas, siendo inversamente proporcional al grado de reactivación. Finalmente, para evaluar la influencia de diferentes sistemas de propulsión, o tipos de arma en el que estos proyectiles fueron empleados, se realizó un análisis PLS sobre la variabilidad morfológica entre las coordenadas de forma y el peso de las puntas de proyectil.

\section{Resultados}

La clasificación tipológica inicial permitió fraccionar la muestra según la forma de su área de enmangue, entre pedunculadas $(n=44) \mathrm{y}$ apedunculadas $(n=87)$. El peso promedio de las puntas es de 10,8 g, estando la mayor parte de los especímenes dentro de la franja entre los 4 y $20 \mathrm{~g}(n=114 ; 87,5 \%)$. Aproximadamente el $5 \%(n=7)$ pesan más de $20 \mathrm{~g}$ y el $7,5 \%$ $(n=10)$ menos de $4 \mathrm{~g}$ (Tabla 1$)$. El adelgazamiento bifacial es la técnica de talla más utilizada, representado casi el $97 \%$ de la muestra $(n=$ 127). Solo cuatro artefactos, confeccionados en basalto (representando el 3,1\% de la muestra), fueron realizados mediante reducción bifacial (Aschero y Hocsman 2004). La materia prima con mayor presencia en el registro es el cuarzo $(n=60)$, que representa el 45,8\% de la muestra. El resto del conjunto se dividen en: sílice microcristalino $(24,4 \% ; n=32)$; calcedonia $(19,8 \% ; n$ $=26)$; basalto $(5,3 \% ; n=7)$; jaspe $(2,3 \% ; n=$ $3)$; ópalo $(0,8 \% ; n=1)$; pórfido $(0,8 \% ; n=1)$ y materia prima indeterminada $(0,8 \% ; n=1)$.

Los resultados del análisis funcional de las puntas de proyectil según el análisis discriminante propuesto por Shott (1997) se resumen en la Tabla Suplementaria 2. Allí se observa que la mayor parte de la muestra analizada formó parte de dardos arrojados con propulsor, mientras que una pequeña fracción ha sido clasificada como puntas de flecha. Si se considera el lapso temporal al que pertenecen las puntas lanceoladas, momento en que el arco no existía, este último subconjunto constituiría una categorización errónea. Una explicación al respecto surgiría de la historia de vida de los artefactos, ya que algunos de ellos fueron afectados por actividades de reactivación, alterando sus dimensiones (i.e., ancho máximo o peso). Además, los ejemplares cuyo peso excede los $20 \mathrm{~g}(n=7)$ podrían haber sido utilizados en lanzas arrojadizas, aunque aún se requiere un estudio específico para considerar el empleo contemporáneo de ambos sistemas (i.e., propulsor y lanza). 

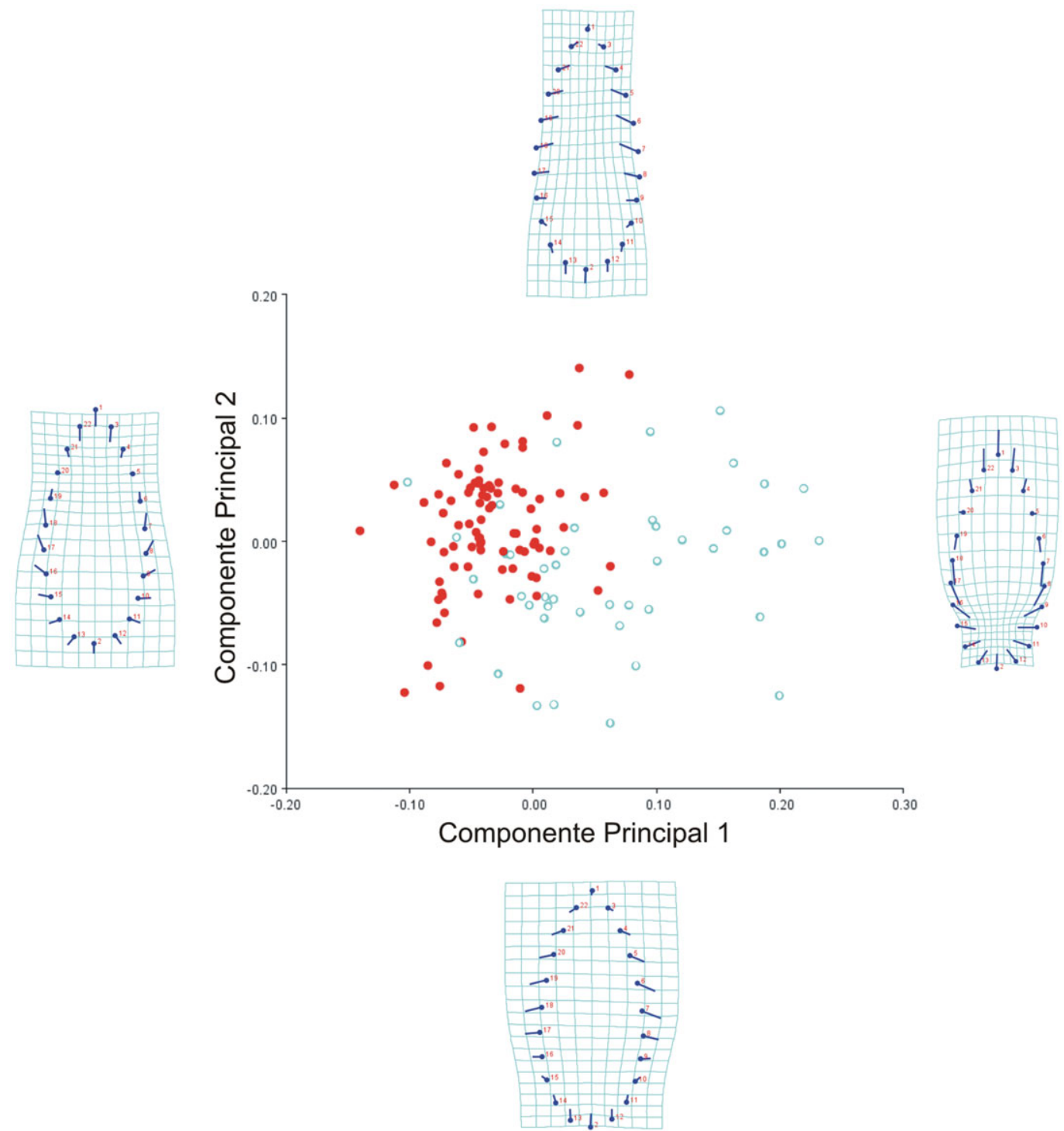

Figura 3. Representación gráfica del análisis de componentes principales (CP1 vs. CP2). Las puntas apendunculadas están representadas por círculos rojos y las pedunculadas por anillos de borde azul. (Color online)

Desde la aplicación de las metodologías de la morfometría geométrica, el análisis de componentes principales indicó que los dos primeros componentes explican el 69,4\% de la variación morfológica, correspondiendo al primer componente el 44,2\% (donde se manifiestan cambios relacionados con la presencia o no de pedúnculo). En la Figura 3 se observa que los ejemplares apedunculados están restringidos en el sector izquierdo del gráfico mientras que los peduncula- dos, si bien se localizan mayoritariamente a la derecha del gráfico, poseen una distribución más amplia, abarcando también el sector de las puntas apedunculadas. Es decir que no existe una clara separación entre los dos tipos de puntas. El segundo componente explica el 25,2\% de la variación, indicando cambios en el alargamiento y ancho de las piezas (Figura 3). Por su parte, el tercer componente principal explica el 7,1\% de la variación morfológica (alcanzando un total de 

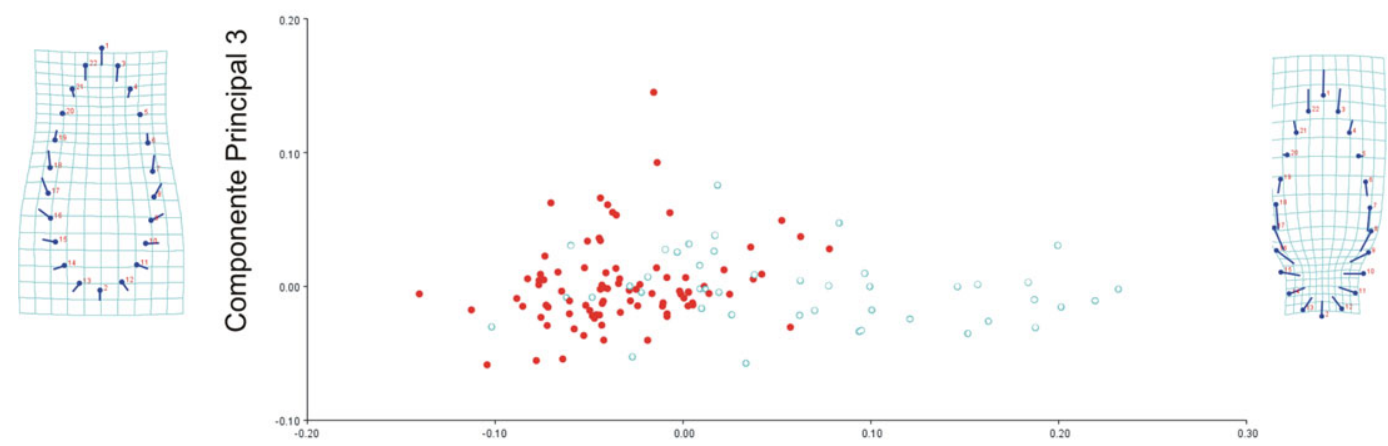

Componente Principal 1

Figura 4. Representación gráfica del análisis de componentes principales (CP1 vs. CP3). Las puntas apendunculadas están representadas por círculos rojos y las pedunculadas por anillos de borde azul. (Color online)

$76,5 \%$ entre los tres componentes principales) y refleja asimetrías posiblemente relacionadas con el efecto de actividades de mantenimiento o reactivación de las puntas (Figura 4).

El test de PLS (Rohlf 1990) indicó una correlación no significativa $(r=0,14 ; p=0,74)$ entre el logaritmo del tamaño centroide y las coordenadas Procrustes, por lo que no existiría una relación entre el tamaño general de la punta y la forma, es decir, los cambios en la forma no se explicarían principalmente por cambios alométricos producidos por reactivación. El análisis de variables canónicas (VC) fue utilizado para observar la influencia de la materia prima en la variación morfológica observada de las puntas (Figura 5). Las dos primeras VC explican el $47,2 \%$ de la variación observada, lo que se corres- ponde con un $26,4 \%$ para la primera variable (VC1) y el 20,8\% restante a la segunda variable (VC2). Los resultados señalan diferencias significativas entre algunas materias primas empleadas y las variables de forma (Tabla 2), especialmente en la calcedonia, sílice, basalto y cuarzo.

La relación entre el lugar de procedencia de los artefactos y la variación morfológica observada se evaluó también mediante un análisis de VC. En este caso, las dos variables canónicas explican el $61,6 \%$ de la variación. La VC1 corresponde al $42,2 \%$ de la muestra, mientras que a la VC2 representa el 19,4\% del total (Figura 6). Se observan diferencias significativas entre algunos lugares de procedencia y las variables de forma (Tabla 3). Las principales diferencias se observan entre los ejemplares provenientes de 
Tabla 2. P-Valores de las Pruebas de Permutación (10.000 Rondas de Permutación) para Distancias Procrustes entre los Grupos, según Materia Prima.

\begin{tabular}{|c|c|c|c|c|c|c|c|}
\hline & Basalto & Calcedonia & Cuarzo & INDET $^{\mathrm{a}}$ & Jaspe & Pórfiro & $\overline{\text { Sílice }}$ \\
\hline Calcedonia & 0.05 & & & & & & \\
\hline Cuarzo & 0.04 & 0.03 & & & & & \\
\hline INDET & 0.07 & 0.09 & 0.07 & & & & \\
\hline Jaspe & 0.08 & 0.07 & 0.09 & 0.09 & & & \\
\hline Pórfiro & 0.11 & 0.09 & 0.10 & 0.13 & 0.1 & & \\
\hline Sílice & 0.03 & 0.03 & 0.03 & 0.07 & 0.06 & 0.1 & \\
\hline Ópalo & 0.09 & 0.07 & 0.08 & 0.11 & 0.1 & 0.08 & 0.08 \\
\hline
\end{tabular}

${ }^{\mathrm{a}}$ INDET $=$ materia prima indeterminada. Los números en negrita corresponden a las correlaciones significativas.

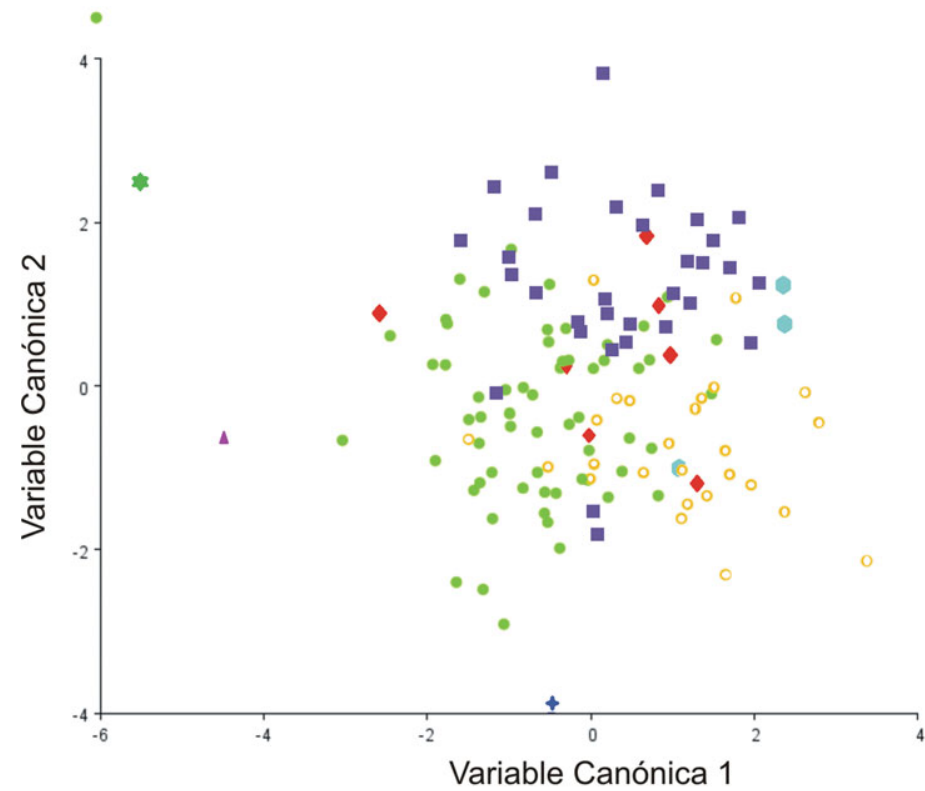

Figura 5. Influencia de la materia prima en la variación morfológica observada. (Color online)

Achala, San Roque, Lavaisse, Sayape, La Iseca y Alto Pelado.

El resultado de la regresión entre las coordenadas de forma y el espesor máximo indicó que no existe una correlación significativa entre las coordenadas de forma y el espesor máximo de las puntas de proyectil $(p=0,13)$. Por su parte el análisis PLS de la relación entre la variación morfológica y el índice de reducción dio una correlación positiva y significativa ( $r=0,37 ; p=0,0007)$. Finalmente, el PLS entre las coordenadas de forma y el peso de las puntas de proyectil, realizado para evaluar si la variabilidad morfológica está influida por la coexistencia de distintos sistemas de armas (i.e., propulsor y lanza), dio una baja correla- ción positiva y no significativa $(r=0,24 ; p=$ $0,11)$.

\section{Discusión de los Resultados}

Las puntas de proyectil con tipologías lanceoladas han sido recuperadas frecuentemente por investigaciones arqueológicas en ambas vertientes de los Andes para latitudes que van, como mínimo, entre $20^{\circ}$ y $36^{\circ}$ S. Es notorio, en esta amplia macrorregión, lo coincidente de su presencia en el registro para finales del Holoceno temprano y parte del Holoceno medio. En este contexto, las puntas Ayampitín fueron el primer conjunto en toda Sudamérica en ser posicionado dentro de una secuencia maestra 
Tabla 3. P-Valores de las Pruebas de Permutación (10.000 Rondas de Permutación) para Distancias Procrustes entre los Grupos, según Procedencia.

\begin{tabular}{|c|c|c|c|c|c|c|c|c|}
\hline & Achala & A. Pelado & C. Sur & San Roque & La Iseca & La Primavera & Lavaisse & P. Sandía \\
\hline A. Pelado & 0.14 & & & & & & & \\
\hline C. Sur & 0.09 & 0.48 & & & & & & \\
\hline San Roque & 0.002 & 0.03 & 0.24 & & & & & \\
\hline La Iseca & 0.08 & 0.33 & 1 & 0.07 & & & & \\
\hline La Primavera & 0.12 & 0.86 & 1 & 0.32 & 1 & & & \\
\hline Lavaisse & 0.04 & 0.5 & 0.40 & 0.04 & 0.28 & 0.61 & & \\
\hline P. Sandía & 0.26 & 0.8 & 0.60 & 0.11 & 0.4 & 1 & 0.77 & \\
\hline Sayape & 0.07 & 0.34 & 0.38 & 0.11 & 0.28 & 0.7 & 0.53 & 0.87 \\
\hline
\end{tabular}

Nota $:$ A. Pelado = Alto Pelado; C. Sur = Cruz del Sur; P. Sandía = Planta de Sandía. Los números en negrita corresponden a las correlaciones significativas.

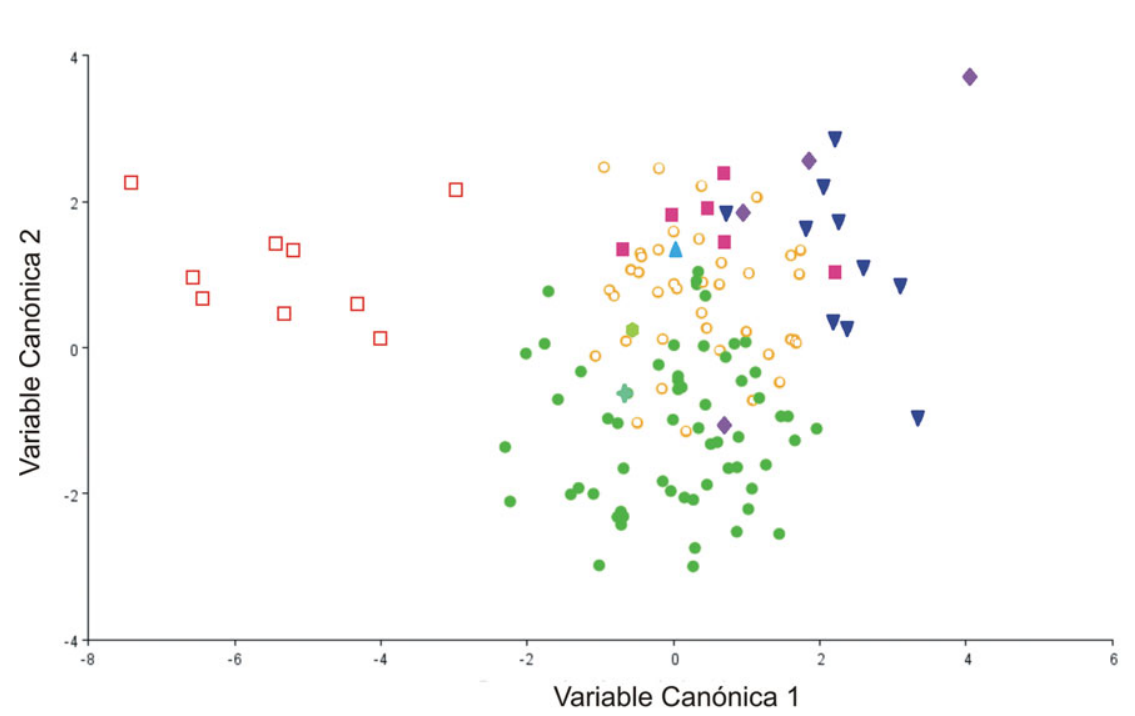

Figura 6. Relación entre el lugar de procedencia de las puntas de proyectil y la variación morfológica observada. (Color online)

(González 1960). Sin embargo, si bien en diferentes sectores de Argentina y Chile proliferaron los estudios sobre cabezales líticos lanceolados, se registraron escasas investigaciones para las puntas Ayampitín. Con la implementación de las metodologías aquí planteadas se describieron y repensaron estos cabezales líticos en dos líneas centrales: el diseño tecnológico del sistema de armas y la implicancia del conjunto analizado dentro de la problemática del poblamiento regional. Finalmente, a partir de los resultados observados, puede inclusive ponerse en discusión la validez del término "Ayampitín" como concepto lo suficientemente abarcativo para describir a las puntas de proyectil lanceoladas del centro de Argentina.
Desde el análisis tecno-morfológico formal, se observa una clara prevalencia del diseño apedunculado en ambas áreas de interés (las Sierras Centrales y el norte de Pampa Seca). La técnica de talla seleccionada para confeccionar los artefactos fue el adelgazamiento bifacial. Son excepciones a este planteo las puntas sobre basaltos recuperadas en el norte de Pampa Seca. Las rocas seleccionadas como soporte presentan características diferentes en cuanto a la predictibilidad de fractura (en especial el cuarzo), aunque todas son de buena o muy buena calidad para la talla. En relación con esto, la disponibilidad en el paisaje regional no es similar en todos los casos: el cuarzo se presenta en el área en forma ubicua, en tanto que las restantes materias 
primas poseen una localización más acotada, encontrándose a distancias superiores a los 50 km de los sitios considerados (Heider et al. 2015). El estudio morfométrico evidenció la existencia de una correlación significativa de algunas de las materias primas con la forma de los ejemplares. Esto es especialmente claro entre los ejemplares provenientes de Achala (donde casi la totalidad de los ejemplares son de cuarzo), los cuales se diferencian notoriamente del resto de las puntas (donde las diferentes variedades de sílices son más comunes). Esto indicaría la existencia de una disponibilidad o acceso diferencial a ciertas materias primas en determinados sectores, influenciando la variabilidad morfológica regional.

El estudio morfométrico indica que la variación morfológica observada puede agruparse en dos morfotipos o variedades principales, lo cual fue sugerido en un estudio realizado previamente con una muestra más pequeña (Rivero y Heider 2017). Los dos componentes principales se discriminan, al igual que se observa con los estudios tecno-tipológicos formales, por la presencia o ausencia de pedúnculo esbozado o destacado. La mayor dispersión se destaca entre las pedunculadas. La variabilidad mencionada podría explicarse, en parte, por la historia de vida de los artefactos (episodios de reactivación y reformatización que influyen más en el limbo que en el pedúnculo) y su posible afectación del diseño original, como lo sugiere el resultado significativo del análisis PLS entre la variación morfológica y el índice de reducción. Sin embargo, la estadística multivariada muestra que no hay una correlación significativa entre el tamaño centroide y las coordenadas Procrustes, descartando un cambio alométrico. Para solucionar esta aparente contradicción se exploró, mediante un análisis PLS, la correlación entre el segundo componente principal (que evidencia cambios en el alargamiento y ancho de las puntas de proyectil) y el índice de reducción de Iriarte (1995), obteniéndose una correlación no significativa $(r=0,12 ; p=0,16)$. Esto indicaría que las formas más pequeñas no serían resultado de la reactivación de un diseño estandarizado representado por las formas más grandes, sino que se trataría de dos tamaños básicos (cada uno de los cuales incluye puntas con y sin pedúnculo) sobre los cuales las actividades de reactivación influyeron de manera paralela.

Los resultados del análisis PLS entre las coordenadas de forma y el peso indican que no existen variaciones morfológicas relacionadas con requerimientos de rendimiento producidos por el empleo de dos sistemas de armas diferentes (i.e., propulsor y lanza). En este sentido, ambos diseños básicos pudieron ser empleados en proyectiles arrojados con propulsor, el cual habría sido el sistema de armas principal empleado durante este período. La mayor parte de las puntas de proyectil $(n=126 ; 96 \%)$ se encuentran en un rango de peso apto para su empleo como puntas de dardos arrojados con propulsor. Asimismo, se abordó la problemática del sistema de armas implementado utilizando el análisis discriminante; esto posibilitó clasificar un alto porcentaje dentro del grupo de puntas de dardo $(n=110 ; 84 \%)$. Por consiguiente, podemos sostener que el sistema de armas empleado por las poblaciones de cazadores-recolectores durante el Holoceno medio fue el propulsor.

\section{Conclusiones y Nuevas Líneas de Investigación}

Las puntas de proyectil lanceoladas constituyen artefactos con características técnicas reconocibles y relativamente acotadas temporalmente en la arqueología del centro, oeste y noroeste de Argentina en general y de las Sierras Centrales en particular. Los abordajes utilizados para su estudio estuvieron ligados, hasta el momento, a su ubicación temporal en contextos arqueológicos, a las cuestiones tecnológicas y a la estadística tradicional. En este trabajo se utilizó la morfometría geométrica para caracterizar esta clase de artefacto cuyos resultados representan, entendemos, un punto de partida para abordajes de mayor complejidad utilizando una mayor cantidad de datos.

Un primer aspecto de interés es la influencia de las materias primas utilizadas sobre la morfología. Las rocas seleccionadas como soporte tienen características diferentes, principalmente en la predictibilidad de su fractura. No puede afirmarse, a partir de la muestra analizada, la existencia de una elección de rocas particulares para la confección de los diseños de puntas. Más 
aún, el estudio morfométrico refuerza la idea de que la selección de materia prima estaría, en muchos casos, ligada a sus aptitudes y disponibilidad más que a otros factores de índole social o identitario (Speth et al. 2013).

La separación entre puntas pedunculadas y apedunculadas, obvia a nivel macroscópico, fue corroborada por el estudio morfométrico, señalando asimismo una gran variabilidad. Una perspectiva a desarrollar estará orientada a comprobar la posibilidad de que la presencia o ausencia de pedúnculo está ligada al diseño de proyectiles con requerimientos funcionales diferentes para la caza de presas a larga distancia en espacios abiertos o, de forma inversa, en sectores acotados del paisaje, entre otras posibilidades. En relación con esto, la inclusión de la mayor parte de los especímenes dentro de un sistema de armas con el uso de propulsor es sustentada tanto por los resultados obtenidos como por la ubicación temporal del conjunto analizado, momento para el cual el arco y la flecha no eran innovaciones tecnológicas presentes.

Por otra parte, los resultados obtenidos aportan información para la discusión de los modelos de poblamiento regional actualmente vigentes. En este sentido, en un escenario riesgoso como lo puede ser el proceso de poblamiento de un nuevo espacio, se espera que se incrementen las innovaciones tecnológicas por variación guiada (sensu Boyd y Richerson 1985). Este consiste en un mecanismo de transmisión cultural donde las innovaciones tecnológicas se basan en un modelo que luego es replicado y modificado por medio de ensayo y error para adaptarlo a las necesidades de sus usuarios. Estas modificaciones podrían generar una gran variabilidad dentro de un determinado diseño de artefactos - por ejemplo, en las puntas de proyectil. Un modelo con estas características fue utilizado por Cardillo (2002) para explicar la transmisión cultural y persistencia diferencial de rasgos en San Antonio de los Cobres (Provincia de Salta, Argentina). Los resultados obtenidos en este trabajo permiten, sobre la base de la variabilidad observada, plantear inicialmente un modelo similar en la región y reforzar la hipótesis de que existió al menos un segundo proceso de exploración y colonización del centro de Argentina diferente al realizado inicialmente en el tardiglaciar. Este habría ocu- rrido desde finales del Holoceno temprano y estuvo integrado a un proceso macrorregional que incluyó al menos el centro-oeste argentino y el centro de Chile (Cortegoso 2014; García 2010; Jackson et al. 2011; Rivero 2012). En este sentido, para avanzar sobre las diferentes etapas de exploración, colonización y ocupación efectiva de la llanuras y sierras pampeanas de Argentina debemos profundizar en conocimientos y análisis combinado de aspectos biogeográficos, paleoambientales, ideológicos y económicos, los cuales sin duda influyeron en la toma de decisiones por parte de los cazadores-recolectores.

Finalmente, la aplicación de la metodología utilizada para las puntas de proyectil permitió caracterizarlas con un importante grado de detalle (algo inédito para la región), asignarlas a sistemas de armas específicos e incluirlas dentro del contexto macrorregional de poblamiento, tal como se ha efectuado para otras latitudes (Martínez 2003; Ratto 2006; Restifo 2013). Un aspecto relevante que surge de este estudio es el registro de especímenes procedentes de la región pampeana. Esto, sumado a la gran variabilidad de puntas registrada, genera la duda sobre la pertinencia del término "Ayampitín" para describir los cabezales líticos lanceolados de las sierras y llanuras pampeanas de Argentina. En este sentido, el trabajo constituye un punto de partida para repensar la validez de Ayampitín como un grupo tipológico cerrado. Al respecto, futuros estudios podrían permitir discriminar subconjuntos o nuevos tipos con rasgos tecnológicos y ubicación geográfico-temporal específica.

Agradecimientos. Queremos agradecer a Silvia Ledda por permitirnos analizar las piezas de museo incluidas en este análisis y a Sebastián Pastor por la gestión realizada con tal fin. A las direcciones de patrimonio de las provincias de San Luis y Córdoba por los permisos otorgados para realizar las investigaciones. A Ivan Perez por la posibilidad de enseñarnos a utilizar técnicas de la morfometría geométrica y estadística multivariada. A Marcelo Cardillo por sus sugerencias y ayuda en la búsqueda bibliográfica. A nuestros compañeros del Área de Arqueología del I.E.H. y el Departamento de Geología de la Universidad Nacional de San Luis. Esta investigación fue financiada por CONICET (PIP 112200801-02678). Finalmente, queremos agradecer a los cinco evaluadores de este trabajo, cuatro anónimos y el Profesor Michael Shott, quienes realizaron valiosas observaciones, las cuales contribuyeron en mejorar aspectos formales, descriptivos y analíticos del manuscrito original. Las ideas vertidas en este trabajo son exclusiva responsabilidad de los autores. 
Declaración de Disponibilidad de Datos. Las colecciones estudiadas en este artículo se encuentran disponibles en el Museo Numba-Charaba de la Ciudad de Carlos Paz, Provincia de Córdoba, Argentina, y en la Cátedra de Prehistoria y Arqueología de la Facultad de Filosofía y Humanidades de la Universidad de Nacional de Córdoba, Argentina. Las bases de datos pueden ser solicitados a los autores de este trabajo mediante el contacto proporcionado.

Material Suplementario. Para acceder a los materiales suplementarios que acompañan este artículo visitar: https://doi.org/10.1017/laq.2018.20.

Tabla Suplementaria 1. Fuentes Probadas y Potenciales de Materias Primas Líticas de uso Arqueológico en las Sierras Centrales.

Tabla Suplementaria 2. Clasificación Funcional de la Muestra Analizada según el Análisis Discriminante propuesto por Shott (1997) y el Peso de las Puntas de Proyectil.

\section{Referencias Citadas}

Aschero, Carlos

1975 Ensayo para una clasificación morfológica de artefactos líticos. Manuscrito en archivo, informe presentado al Consejo Nacional de Investigaciones Científicas y Técnicas (CONICET), Buenos Aires.

1988 De punta a punta: Producción, mantenimiento y diseño en puntas de proyectil precerámicas de la Puna argentina. Actas del IX Congreso Nacional de Arqueología Argentina, pp. 219-229. Facultad de Filosofía y Letras, Universidad de Buenos Aires, Buenos Aires.

Aschero, Carlos y Salomón Hocsman

2004 Revisando cuestiones tipológicas en torno a la clasificación de artefactos bifaciales. En Temas de arqueología. Análisis lítico, editado por Alejandro Acosta, Daniel Loponte y Mariano Ramos, pp. 7-25. Universidad Nacional de Luján, Luján, Argentina.

Aschero, Carlos, Salomón Hocsman y Norma Ratto

2011 Las puntas de proyectil en "mandorla" de Inca Cueva 7: Caracterización tipológica e historia de vida (Puna de Jujuy, Argentina). Estudios atacameños 41:5-28.

Balbuena, José, Miguel González, Fernando Angiolini y Miguel Albero

1982 Presencia humana en la Salina del Bebedero (provincia de San Luis, República Argentina), durante el Holoceno temprano: Su significado paleoclimático. Primera reunión de ciencias del hombre en zonas áridas, pp. 1-14. Universidad Nacional de Cuyo y CRICYTME, Mendoza, Argentina.

Bárcena, Roberto

2001 Prehistoria del centro-oeste argentino. En Historia argentina prehispánica, editado por Eduardo Berberián y Axel Nielsen, Tomo II, pp. 561-634. Editorial Brujas, Córdoba, Argentina.

Berberián, Eduardo y Horacio Calandra

1984 Investigaciones arqueológicas en El Peñoncito, San Juan, República Argentina. Revista del Museo de La Plata VIII(56):139-169.

Bettinger, Robert, James O'Connell y Thomas David

1991 Projectile Points as Time Markers in the Great Basin. American Anthropologist 93(1):166-172.
Bookstein, Fred

1982 Foundations of Morphometrics. Annual Review of Ecology and Systematics 13:451-470.

1991 Morphometric Tools for Landmark Data: Geometry and Biology. Cambridge University Press, Cambridge.

1997 Landmark Methods for Forms Without Landmarks: Localizing Group Differences in Outline Shape. Medical Image Analysis 1(3):225-243.

Boyd, Richard y Peter Richerson

1985 Culture and the Evolutionary Process. University of Chicago Press, Chicago.

Capriles, José y Juan Albarracín-Jordán

2013 The Earliest Human Occupation in Bolivia: A Review of the Archaeological Evidence. Quaternary International 301:46-59.

Cardillo, Marcelo

2002 Transmisión cultural y persistencia diferencial de rasgos. Un modelo para el estudio de la variación morfológica de las puntas de proyectil lanceoladas de San Antonio de los Cobres, Provincia de Salta, Argentina. En Perspectivas integradoras entre arqueología y evolución, editado por Gustavo Martínez y José Lanata, pp. 97-119. INCUAPA, Universidad Nacional del Centro de la Provincia de Buenos Aires, Olavarría, Argentina.

2006 Explorando la variación en las morfologías líticas a partir de la técnica de análisis de contornos. El caso de las puntas de proyectil del Holoceno medio-tardío de la Puna de Salta (San Antonio de los Cobres, Argentina). Werkén 7(2):77-88.

2010 Some Applications of Geometric Morphometrics to Archaeology. En Morphometrics for Nonmorphometricians, editado por Ashraf Elewa, pp. 235-241. Lecture Notes in Earth Sciences Vol. 124. SpringerVerlag, Berlín.

Cardillo, Marcelo y Judith Charlín

2010 Diversificación morfológica en las puntas de proyectil de Patagonia. Explorando dimensiones espaciales y temporales. II Encuentro de Morfometría Geométrica y Estudios Filogenéticos, p. 7. Universidad Nacional de La Plata, La Plata, Argentina.

Castiñeira, Carola, Marcelo Cardillo, Judith Charlin y Jorge Baeza

2011 Análisis de morfometría geométrica en puntas cola de pescado del Uruguay. Latin American Antiquity 22:335-358.

Cattáneo, Roxana, Andrés Izeta y Thiago Costa

2013 El patrimonio arqueológico de los espacios rurales de la provincia de Córdoba. Universidad Nacional de Córdoba, Córdoba, Argentina.

Cattáneo, Roxana, Andrés Izeta y José Caminoa

2016 A Fishtail Projectile Point from the Southern Pampean Hills, Characato, Córdoba, Argentina. PaleoAmerica 2(3):274-276.

Charlin, Judith y Rolando Gonzalez-José

2012 Size and Shape Variations in Late Holocene Projectile Points from Southern Patagonia: A Geometric Morphometric Study. American Antiquity 77:221-242.

Cornero, Silvia, Walter Neves y Diego Rivero

2014 Nuevos aportes a la cronología de las ocupaciones tempranas en las Sierras de Córdoba. La gruta de Candonga (Córdoba, Argentina). Relaciones de la Sociedad Argentina de Antropología XXXIX(1):285-292.

Cortegoso, Valeria

2005 Mid-Holocene Hunters in the Andes Mountains: Environment, Resources and Technological Strategies. Quaternary International 132(1):71-80. 
2014 Valle de las Taguas, ARQ-18: Estratigrafía, secuencia temporal y ocupaciones humanas. En Arqueología de ambientes de altura de Mendoza y San Juan (Argentina), editado por Valeria Cortegoso, Victor Durán y Alejandra Gasco, pp. 209-242. EDIUNC, Mendoza, Argentina.

De Souza, Patricio

2006 Los sistemas de proyectiles durante el proceso Arcaico Tardío-Formativo Temprano de la Puna de Atacama: Una aproximación desde el análisis de las puntas de proyectil de quebrada Tulán. Tesis de maestría inédita, Instituto de Investigaciones Arqueológicas y Museo, Universidad de Tarapacá; Departamento de Antropología, Universidad Católica del Norte, San Pedro de Atacama, Chile.

Demaría, Manuel

2008 Cambios en la cobertura de pastizales pampeanos semiáridos en San Luis (Argentina), y su relación con variables climáticas y ambientales. Análisis multitemporal mediante teledetección y SIG. Tesis doctoral inédita, Departamento de Geología, Geografía y Medio Ambiente, Universidad de Alcalá de Henares, Madrid.

Durán, Víctor

1997 Arqueología del valle del Río Grande, Malargüe, Mendoza. Tesis doctoral inédita, Facultad de Ciencias Naturales y Museo, Universidad Nacional de La Plata, La Plata, Argentina.

Ebert, James

1992 Distributional Archaeology. University of New Mexico Press, Albuquerque.

Ericson, Jonathon

1984 Toward the Analysis of Lithic Production Systems. En Prehistoric Quarries and Lithic Production, editado por Jonathon Ericson y Barbara Purdy, pp.1-9. Cambridge University Press, Cambridge.

Franco, Nora, Alicia Castro, Marcelo Cardillo y Judith Charlín

2009 La importancia de las variables morfológicas, métricas y de microdesgaste para evaluar las diferencias en diseños de puntas de proyectil bifaciales pedunculadas: Un ejemplo del sur de Patagonia continental. Magallania 37(1):99-112.

Fenenga, Franklin

1953 The Weights of Chipped Stone Points: A Clue to Their Functions. Southwestern Journal of Anthropology 9(3):309-323.

Gambier, Mariano

1974 Horizonte de cazadores tempranos en los Andes Centrales argentino-chilenos. Нипис-Hиаr 2:43-103.

García, Alejandro

2003 La ocupación temprana de los Andes Centrales argentinos (ca. 11.000-8.000 años C14 AP). Relaciones de la Sociedad Argentina de Antropología XXVIII:153165.

2005 Human Occupation of the Central Andes of Argentina $\left(32^{\circ}-34^{\circ} \mathrm{S}\right)$ during the Mid-Holocene. Quaternary International 132(1):61-70.

2010 Human Occupation during the Mid-Holocene in Western Argentina. Current Anthropology 51(3):415416.

González, Alberto Rex

1952 Antiguo horizonte precerámico en las Sierras Centrales de la Argentina. Runa V:110-133.

1960 La estratigrafía de la gruta de Intihuasi (Prov. de San Luis, R.A.) y sus relaciones con otros sitios precerámicos de Sudamérica. Revista del Instituto de Antropología 1:5-296.

Gunz, Philipp y Philipp Mitteroecker

2013 Semilandmarks: A Method for Quantifying Curves and Surfaces. Hystrix, the Italian Journal of Mamma$\operatorname{logy} 24(1): 103-109$.

Gradín, Carlos

1984 Investigaciones arqueológicas en Casa de Piedra. Informe en archivo, Dirección General de Cultura y Ente Ejecutivo Casa de Piedra, La Pampa, Argentina.

Heider, Guillermo

2015 Los pueblos originarios en el norte de Pampa Seca. Una mirada arqueológica a los cazadores-recolectores del sur de las provincias de Córdoba y San Luis, Argentina. Tesis doctoral inédita, Escuela de Historia, Facultad de Filosofía y Humanidades, Universidad Nacional de Córdoba, Córdoba, Argentina.

Heider, Guillermo y Rafael Curtoni

2016 Investigaciones arqueológicas en la Provincia de San Luis: A 150 años de sus inicios, historia y perspectivas. Revista del Museo de Antropología 9(1):35-48.

Heider, Guillermo y Alejandro Demichelis

2015 Loma de los Pedernales, a Local Raw Material Source in the North of Pampa Seca, Argentina. Quaternary International 375:3-12.

Heider, Guillermo, Diego Rivero y Edgardo Baldo

2015 Rocas de uso arqueológico en Sierras Centrales. Fuentes de recursos líticos identificadas y potenciales en las provincias de Córdoba y San Luis, Argentina. Revista de antropología del Museo de Entre Ríos 1(2):55-72.

Heider, Guillermo, Ariel Ortiz Suarez, Ernesto Perino, Jorge Orlando Chiesa, Raúl Andrés Gil, Cristian Bazán y Alberto Basaez

2017 Análisis multi-proxy para el estudio en canteras de rocas silíceas y movilidad de cazadores-recolectores en el norte de Pampa Seca. Actas del VIII Congreso de Arqueología de la Región Pampeana Argentina, pp. 8586. Luján, Argentina.

Hocsman, Salomón

2010 Cambios en las puntas de proyectil durante la transición de cazadores-recolectores a sociedades agropastoriles en Antofagasta de la Sierra (Puna argentina). Arqueología 16:59-86.

Hoguin, Rodolph y Hugo Yacobaccio

2012 Análisis lítico de ocupaciones del Holoceno medio de Hornillos 2 (Jujuy, Argentina): Discutiendo la tecnología y distribución de las puntas de proyectil "San Martín". Chungara 44(1):85-99.

Hughes, Susan

1998 Getting to the Point: Evolutionary Change in Prehistoric Weaponry. Journal of Archaeological Method and Theory 5(4):345-408.

Iriarte, Javier

1995 Afinando la puntería: Tamaño, forma y rejuvenecimiento en las puntas de proyectil pedunculadas del Uruguay. Arqueología del Uruguay. VIII Congreso Nacional de Arqueología Uruguaya, pp. 142-151. Montevideo.

Jackson, Donald, Antonio Maldonado, Matthieu Carré y Roxana Seguel

2011 Huentelauquén Cultural Complex: The Early Peopling of the Pacific Coast in the South-America Southern cone. En Peuplements et préhistoire en Amériques, editado por Denis Vialou, pp. 221-232. Éditions du Comité des Travaux Historiques et Scientifiques, Paris. 
Knecht, Heidi

1997 Projectile Points of Bone, Antler and Stone. Experimental Explorations of Manufacture and Use. En Projectile Technology, editado por Heidi Knecht, pp. 191-212. Plenum Press, New York.

Klingenberg, Cris

2009 MorphoJ. The University of Manchester. http:// flywings.org.uk/MorphoJ.page.htm, accedido el 15 de marzo de 2017.

Klingenberg, Cris y Leandro Monteiro

2005 Distances and Directions in Multidimensional Shape Spaces: Implications for Morphometric Applications. Systematic Biology 54(4):678-688.

Laguens, Andrés, Eduardo Pautassi, Gisela Sario y Roxana Cattáneo

2007 ELS1, a Fishtail Projectile Point Site from Central Argentina. Current Research in the Pleistocene 24:5557.

Martínez, Jorge

2003 Ocupaciones humanas tempranas y tecnología de caza en la microrregión de Antofagasta de la Sierra (10.000-7.000 AP). Tesis doctoral inédita, Facultad de Ciencias Naturales e Instituto Miguel Lillo, Universidad Nacional de Tucumán, Tucumán, Argentina.

2007 Ocupaciones humanas tempranas y tecnología de caza en Antofagasta de la Sierra, Puna Meridional argentina (10.000-7.000 AP). Cazadores-recolectores del Cono Sur. Revista de arqueología 2:129-150.

Mitteroecker, Philipp y Fred Bookstein

2011 The Ontogenetic Trajectory of the Phenotypic Covariance Matrix, with Examples from Craniofacial Shape in Rats and Humans. Evolution 63(3):727-737.

Mitteroecker, Philipp y Philipp Gunz

2009 Advances in Geometric Morphometrics. Evolutionary Biology 36:235-247.

Neme, Gustavo y Adolfo Gil

2009 Human Occupation and Increasing Mid-Holocene Aridity. Current Anthropology 50(1):149-163.

Núñez, Lautaro, Martín Grosjean e Isabel Cartajena

2005 Ocupaciones humanas y paleoambientes en la Puna de Atacama. Universidad Católica del Norte y Taraxacum, San Pedro de Atacama, Chile.

Okumura, Mercedes y Astolfo Araujo

2014 Long-Term Cultural Stability in Hunter-Gatherers: A Case Study Using Traditional and Geometric Morphometric Analysis of Lithic Stemmed Bifacial Points from Southern Brazil. Journal of Archaeological Science 45:59-71.

Pastor, Sebastián, Eduardo Pautassi y Diego Rivero

2005 Los sistemas de armas de las comunidades agroalfareras de Córdoba: Una aproximación arqueológica y experimental. Actas del XIII Congreso Nacional de Arqueología Argentina, Tomo 4, pp. 253-266. Editorial Brujas, Córdoba, Argentina.

Pautassi, Eduardo

2011 Tecnología de proyectiles, durante el Holoceno temprano, en la porción austral de las sierras pampeanas. En Armas prehispánicas: Múltiples enfoques para su estudio en Sudamérica, editado porMartínez Jorge y Damian Bozzuto, pp. 115-134. Fundación de Historia Natural Félix de Azara, Buenos Aires.

Perez, Ivan, Valeria Bernal y González Paula

2006 Differences Between Sliding Semi-Landmark Methods in Geometric Morphometrics, with an Application to Human Craniofacial and Dental Variation. Journal of Anatomy 16:769-784.
Pintar, Elizabeth

2004 Cueva Salamanca 1: Ocupaciones altitermales en la Puna Sur (Catamarca). Relaciones de la Sociedad Argentina de Antropología XXIX:357-366.

Politis, Gustavo, Pablo Messineo y Cristian Kaufmann

2004 El poblamiento temprano de las llanuras pampeanas de Argentina y Uruguay. Complutum 15:207-224.

Ratto, Norma

2003 Estrategias de caza y propiedades del registro arqueológico en la Puna de Chaschuil (Departamento de Tinogasta, Catamarca, Argentina). Tesis doctoral inédita, Facultad de Filosofía y Letras, Universidad de Buenos Aires, Argentina.

2006 El Arcaico y Formativo en la puna de Chaschuil a través del diseño de las puntas líticas (departamento de Tinogasta, Catamarca). Cazadores-recolectores del Cono Sur. Revista de Arqueología 1:93-109.

Restifo, Federico

2013 Tecnología de caza durante el Holoceno temprano y medio en la puna de la Provincia de Salta (República Argentina): Patrones de variación y procesos de cambio. Comechingonia 17:59-84.

Rivero, Diego

2009 Ecología de cazadores-recolectores del sector central de las Sierras de Córdoba (Rep. Argentina). BAR International Series 2007, Oxford.

2012 La ocupación humana durante la transición Pleistoceno-Holoceno (11,000-9,000 a.P.) en las Sierras Centrales de Argentina. Latin American Antiquity 23:551-564.

Rivero, Diego y Guillermo Heider

2016 Estrategias de caza durante el Holoceno medio en las pampas de altura de las sierras de Córdoba. Actas del XIX Congreso Nacional de Arqueología Argentina, pp. 3085-309. Tucumán, Argentina.

2017 Morfometría geométrica en puntas de proyectil lanceoladas de las sierras centrales (prov. de Córdoba y San Luis). Implicancias para la discusión del poblamiento regional. Revista del Museo de Antropología 1:7582.

Rivero, Diego, Guillermo Heider y Pastor Sebastián

2015 Identificación de una punta cola de pescado en las Sierras de Córdoba. Implicancias para el poblamiento del centro de Argentina. Cuadernos del Instituto de Antropología y Pensamiento Latinoamericano 24(1):151-155.

Rondeau, Michael

1996 When Is an Elko? En Stone Tools: Theoretical Insights into Human Prehistory, editado por George Odell, pp. 229-243. Plenum Press, New York.

Rohlf, James

1990 Rotational Fit (Procrustes) Methods. En Proceedings of the Michigan Morphometrics Workshop, editado por James Rohlf y Fred Bookstein, pp. 227236. Museum of Zoology, University of Michigan, Ann Arbor.

2004a Relative Warps, v. 1.26. Department of Ecology and Evolution, State University of New York, Stony Brook. Electronic document, http://life.bio.sunysb.edu/ morph/, accedido el 15 de Marzo de 2017.

2004b Relative Warps, v. 1.4. Department of Ecology and Evolution, State University of New York, Stony Brook. Electronic document, http://life.bio.sunysb.edu/ morph/, acedido el 15 de Marzo de 2017.

2014c Relative Warps, v. 1.54. Department of Ecology and Evolution, State University of New York, Stony 
Brook. Electronic document, http://life.bio.sunysb.edu/ morph/, accedido el 15 de Marzo de 2017.

Rohlf, James y Marco Corti

2000 The Use of Two-Block Partial Least Squares to Study Covariation in Shape. Systematic Biology 49(4):740-753.

Rohlf, James y Dennis Slice

1990 Extensions of the Procrustes Method for the Optimal Superimposition of Landmarks. Systematic Zoology 39(1):40-59.

Sario, Gisela

2008 Tecnología bifacial en las Sierras de San Luis y depresión del Conlara (Provincia de San Luis, República Argentina) en el Holoceno Temprano. Arqueoweb, Revista sobre arqueología en internet 10:1-18.

2011 Poblamiento humano en la provincia de San Luis: Una perspectiva arqueológica a través del caso de la organización de la tecnología en Estancia La Suiza. Tesis doctoral inédita, Facultad de Filosofía y Humanidades, Universidad Nacional de Córdoba, Córdoba, Argentina.

Shott, Michael J

1997 Stones and Shafts Redux: The Metric Discrimination of Chipped-Stone Dart and Arrow Points. American Antiquity 62:86-101.

Speth, John, Khori Newlander; Andrew White, Ashley Lemke y Lars Anderson

2013 Early Paleoindian Big-Game Hunting in North America: Provisioning or Politics? Quaternary International 285:111-139.
Thomas, David

1978 Arrowheads and Atlatl Darts: How the Stone Got the Shafts. American Antiquity 43:461-472.

Torcida, Sebastián e Ivan Perez

2012 Análisis de Procrustes y el estudio de la variación morfológica. Revista argentina de antropología biológica 14(1):131-141.

Tripaldi, Alfonsina y Steve Forman

2007 Geomorphology and Chronology of Late Quaternary Dune Fields of Western Argentina. Palaeogeography, Palaeoclimatology, Palaeoecology 51(2):300320.

Zelditch, Miriam, Donald Swiderski, David Sheets y William Fink

2004 Geometric Morphometrics for Biologists: A Primer. Academic Press, Cambridge.

\section{Nota}

1. La solución de una variable propuesta por Shott (1997) utiliza las siguientes funciones: a) Dardo: 1,40 (ancho máximo) - 16,85; b) Flecha: 0,89 (ancho máximo) - 7,22. La función que de mayor resultado indica la asignación funcional correcta.

Submitted June 20, 2017; Revised January 17, 2018; Accepted March 22, 2018 